\title{
An Overview of Solar Energy in Chile
}

\author{
Marco Rivera, Patrick Wheeler
}

\begin{abstract}
This paper shows the installed power capacity of conventional and non-conventional renewable energy in the electrical system networks found in Chile, as well as the evolution of the photovoltaic solar capacity installed from 2015 until the present day and the percentage distribution of Non-Conventional Renewable Energy (NCRE) sources. The ten most important solar plants (capacities ranging from $101 \mathrm{MW}$ to $196 \mathrm{MW}$ ) located in the north of the country will be presented, along with those approved or under construction. Together these new plants will represent an increase of $P V$ sources in the energy matrix of 6.625 MW. Finally, technical characteristics of the largest photovoltaic solar plants in Latin America are described, including the 776.230 solar panels installed and covering an area of $2.800 .000 \mathrm{~m}^{2}$ in the Atacama Region.
\end{abstract}

Keywords-Photovoltaic, renewable energy, solar energy.

\section{INTRODUCCIÓN}

La energía solar todavía ocupa una parte pequeña de la producción global de electricidad, pero su función está creciendo rápidamente [1]. Además, en los últimos años ha ganado popularidad no solo gracias a diversas medidas de apoyo gubernamentales, sino también por los resultados esenciales de su implementación, a saber, la madurez tecnológica de la industria y, en algunos casos, la competitividad económica [2].

Dentro de los beneficios de la energía solar, están el que es más barata, ya que tras la inversión inicial, la producción y mantenimiento son mucho más económicos. La energía solar proporciona a la humanidad un recurso energético puro, vasto, duradero y respetuoso con el medio ambiente [3]. Por cada $100 \mathrm{~kW}$ de potencia solar instalada se evita la emisión de $75.000 \mathrm{~kg}$ de $\mathrm{CO}_{2}$ al año [4]. La energía solar está disponible a nivel global, aunque Chile es unos de los territorios con mayor cantidad de horas de sol durante el día, por lo que es fácil y conveniente aprovechar la radiación solar para generar energía. Por otra parte, el mantenimiento es fácil, conveniente $\mathrm{y}$ es posible generar electricidad en sitios remotos [5].

La electricidad se puede generar utilizando energía solar utilizando dos tecnologías diferentes: sistemas fotovoltaicos (PV) y solares concentrados o termosolares (CSP) [6], [7]. Los sistemas solares fotovoltaicos y térmicos solares se han convertido en soluciones altamente competitivas para aplicaciones comerciales, industriales y residenciales como fuente independiente [8].

Hoy en día, el desierto de Atacama en Chile es considerado uno de los lugares del planeta con mayor potencial como fuente energética solar. Esta zona se caracteriza por ser la

Marco Rivera. Director del Laboratorio de Conversión de Energías y Electrónica de Potencia, Universidad de Talca, Curicó, Chile.

Patrick Wheeler. Power Electronics, Machines and Control Group, The University of Nottingham, Nottingham, UK.

978-0-7381-3333-1/21/\$31.00 @2021 IEEE más árida y con mayor radiación del mundo, presentando un alto número de días despejados (sin nubes) en el año, lo que la ha convertido en un escenario perfecto para generar energía solar fotovoltaica a gran escala [9], [10]. Los cielos despejados y limpios del desierto constituyen una característica climática que lo convierte en una de las zonas de la Tierra con mejor capacidad para aprovechar la energía solar [11].

A la fecha, Chile registra una generación total instalada de 24.236 MW, de los cuales un $23 \%$, es decir 5.574,28 MW, proviene de Energías Renovables No Convencionales (ERNC).

Esto se ha logrado seis años antes de la estipulado con la promulgación de la Ley 20.698 en 2013, donde se estableció que para el 2025 el $20 \%$ de la energía debe provenir de estas fuentes. Un 11,20\% del total, o un 48,70\% de la ERNC, es decir 2.714,67 MW, corresponde concretamente a la energía solar fotovoltaica (FV) [12].

Este artículo muestra la capacidad instalada de energía en los sistemas eléctricos que componen la matriz energética con el fin de dar a aquellos académicos e investigadores que se inician en el estudio de energía solar un resumen de la situación actual y sus desafíos.

La distribución porcentual de ERNC, ver Figura 1, se compone principalmente de energía solar, eólica, biomasa, minihidráulica y geotérmica, destacando el predominio de la energía solar fotovoltaica, que se muestra en la zona amarilla del gráfico, con 2.586,11 MW. La Figura 2 muestra la evolución en el tiempo de la capacidad instalada de energía solar fotovoltaica en Chile, desde 2015 hasta la actualidad. Asimismo, la Tabla I detalla las principales características de las 10 plantas solares más grandes del país, según la Comisión Nacional de Energía (CNE). Todos ellos superan los 100 MW y están ubicados en el norte del país, que tiene las tasas de

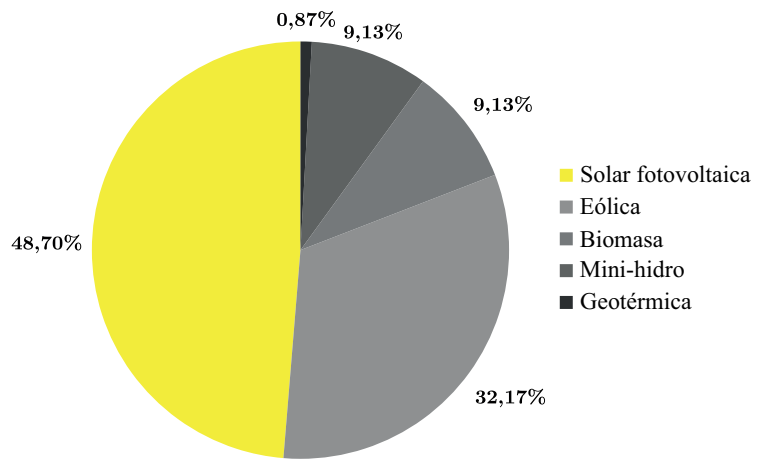

Fig. 1: Capacidad instalada de ERNC en Chile actualizada a marzo de 2020. 


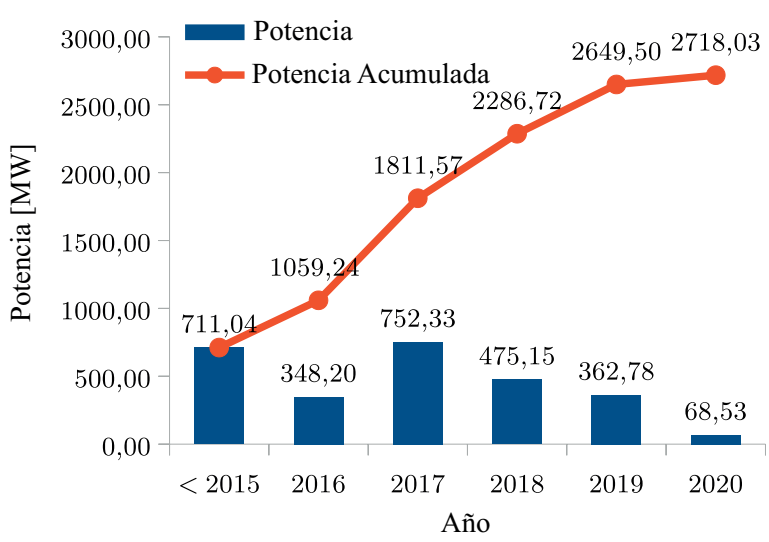

Fig. 2: Capacidad instalada de energía solar fotovoltaica desde 2015 hasta marzo de 2020.

radiación más altas del mundo, como se muestra en el mapa de irradiación horizontal global, Figura 3.

Además, se describen las características técnicas más relevantes de la mayor planta fotovoltaica de Latinoamérica, el parque solar El Romero de ACCIONA Energía, ubicado en la Región de Atacama, con una potencia instalada de 196 MW, compuesto por 776.230 paneles solares y ocupando un superficie de $2.800 .000 \mathrm{~m}^{2}$. Finalmente, se presentan los proyectos más relevantes que se encuentran actualmente en clasificación, aprobados y en construcción. Todos juntos representarán un incremento en la matriz energética de $6.625 \mathrm{MW}$.

\section{Energías Renovables en Chile}

Se consideran fuentes de Energía Renovable No Convencional las siguientes: solar (térmica y fotovoltaica), energía eólica, pequeñas centrales hidroeléctricas (centrales de hasta $20 \mathrm{MW}$ ), biomasa, biogás, geotecnia, mareomotriz. A diferencia de las energías convencionales, estas se caracterizan, en primer lugar, por proceder de fuentes que no contaminan el medio ambiente $\mathrm{y}$, en segundo lugar, por ser restauradas a mayor velocidad que el consumo humano. Chile tiene tres sistemas interconectados distribuidos por todo el país, que en su conjunto se denominan matriz energética, que se describen a continuación [13], [14].

\section{II-A. Sistema Eléctrico Nacional (SEN)}

Compuesto por los antiguos Sistemas Interconectado Central (SIC) e Interconectado del Norte Grande (SING). Abarca desde la región de Arica y Parinacota hasta la Isla Grande de Chiloé, tiene una capacidad instalada de red de 24.068 MW. El $23 \%$ de la capacidad instalada corresponde a fuentes renovables $(2,1 \%$ paso minihidroeléctrico, $11,2 \%$ solar, $7,4 \%$ eólica, $2,1 \%$ biomasa y $0,2 \%$ geotérmica), mientras que el $77 \%$ corresponde a fuentes convencionales $(20 \%$ carbón, $18 \%$ gas natural, $14 \%$ hidráulica, $13 \%$ gas y $12 \%$ hidráulica de paso).

\section{II-B. Sistema Eléctrico de Aysén (SEA)}

El SEA produce electricidad para abastecer a la Región de Aysén del General Carlos Ibáñez del Campo. Tiene una

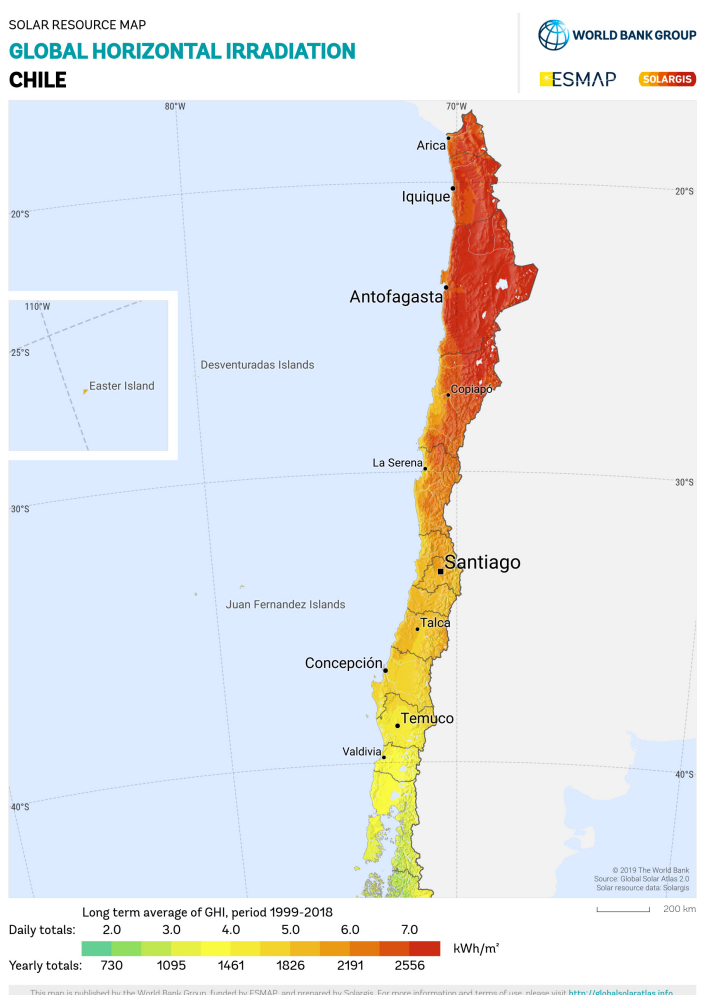

Fig. 3: Mapa global de irradiación horizontal de Chile, Global Solar Atlas.

capacidad instalada de red de $60 \mathrm{MW}$, con $57 \%$ gas, $38 \%$ minihidráulica de pasada y $5 \%$ eólica.

\section{II-C. Sistema Eléctrico de Magallanes (SEM)}

El SEM produce electricidad para abastecer a la Región de Magallanes y la Antártica Chilena. Tiene una capacidad instalada de red de $107 \mathrm{MW}$, con $83 \%$ de gas natural, $15 \%$ de petróleo y $2 \%$ de energía eólica. Como se describió anteriormente, el SEN es el único con fuentes de energía solar fotovoltaica, ya que cubre las regiones del norte del país, que reciben la mayor cantidad de radiación solar promedio diaria en el mundo, lo que le otorga a Chile uno de los mayores potenciales en el uso de esta energía. A pesar de eso, se requiere una diversificación de las fuentes de energía y se deben considerar las siguientes circunstancias [15]:

- Las ERNC tienen variaciones estacionales de generación, es decir, las condiciones invernales no son las mismas que las del verano.

- Hay un aumento de la demanda de energía en los meses de invierno.

- Los parques solares no generan electricidad por la noche.

\section{Situación Actual}

Las disposiciones de la Ley 20.698, establecen que al 2025, el $20 \%$ de la energía debe provenir de fuentes renovables no convencionales. Sin embargo, actualmente, cinco años antes de lo previsto, este porcentaje asciende al $23 \%$, con una gran aportación de la energía solar fotovoltaica. 
TABLA I: Las 10 plantas solares de mayor capacidad conectadas en el SEN. "Capacidad Instalada por Sistema Eléctrico Nacional”, Marzo 2020, CNE

\begin{tabular}{|c|c|c|c|c|c|c|}
\hline Propietario & Nombre & $\begin{array}{l}\text { Puesta en } \\
\text { Servicio }\end{array}$ & Comuna & Región & $\begin{array}{l}\text { Potencia } \\
\text { (MW) }\end{array}$ & Punto de Conexión al SEN \\
\hline Acciona Energía Chile S.A. & Solar El Romero & 2017 & Vallenar & Atacama & 196,00 & Punta Colorada $220 \mathrm{kV}$ \\
\hline Luz del Norte SpA & Luz del Norte & 2016 & Copiapó & Atacama & 141,04 & S/E Carrera Pinto $220 \mathrm{kV}$ \\
\hline Enel Green Power SpA. & Finis Terrae & 2017 & Maria Elena & Antofagasta & 137,31 & Rande $220 \mathrm{kV}$ \\
\hline SunPower Corp. SpA. & El Pelícano & 2018 & La Higuera & Coquimbo & 105,03 & S/E Don Héctor $220 \mathrm{kV}$ \\
\hline María Elena Solar S.A. & Granja Solar & En Pruebas & Pozo almonte & Tarapacá & 105,00 & S/E Lagunas $220 \mathrm{KV}$ \\
\hline Conejo Solar SpA. & Conejo Solar & 2016 & Taltal & Antofagasta & 104,00 & S/E San Francisco $220 \mathrm{kV}$ \\
\hline Chungungo S.A. & Quilapilún & 2017 & Colina & Metropolitana & 103,02 & Polpaico-Los Maquis $220 \mathrm{kV}$ \\
\hline $\begin{array}{l}\text { Austrian Solar Chile } \\
\text { Cuatro Spa }\end{array}$ & Huatacondo & En Prueba & Pozo almonte & Tarapacá & 98,00 & S/E San Simon220KV \\
\hline
\end{tabular}

TABLA II: Parques solares superiores a 100 MW en clasificación, "Proyectos de generación en el SEIA", Marzo 2020, Generadoras de Chile.

\begin{tabular}{|c|c|c|c|c|}
\hline Propietario & Nombre & Comuna & Región & Potencia (MW) \\
\hline Pleiades S.A. & Parque Fotovoltaico Alfa Solar & María Elena & Antofagasta & 726 \\
\hline Colbún S.A. & Planta Fotovoltaica Inti Pacha & María Elena & Antofagasta & 719 \\
\hline Colbún S.A. & Planta Fotovoltaica Jardín Solar & Pozo Almonte & Tarapacá & 556 \\
\hline Andes Solar SPA & Ampliación Parque Fotovoltaico Los Andes & Antofagasta & Antofagasta & 489 \\
\hline Enel Green Power del Sur Spa & Planta Fotovoltaica Sierra Gorda Solar & Sierra Gorda & Antofagasta & 404 \\
\hline Parque Eólico Tal Tal S.A & Parque Fotovoltaico Taltal solar & Taltal & Antofagasta & 317 \\
\hline Golden Sun SpA & Golden Sun & Copiapó & Atacama & 250 \\
\hline Ibereolica Solar Antofagasta SpA. & Proyecto Solar Antofagasta & Antofagasta & Antofagasta & 250 \\
\hline GR Algarrobo SpA & Parque Fotovoltaico Gran Teno & Teno & Maule & 200 \\
\hline Tamarugal Solar SpA. & Parque Fotovoltaico Aurora Solar & Pozo Almonte & Tarapacá & 187 \\
\hline Enel Green Power del Sur Spa & Proyecto Fotovoltaico Lalackama & Taltal & Antofagasta & 181 \\
\hline Tercera Región Solar SpA & Meseta de Los Andes & Los Andes & Valparaiso & 175 \\
\hline Energia Renovable Verano Tres SpA & Parque Solar Fotovoltaico Punta del Viento & La Higuera & Coquimbo & 145 \\
\hline Peldehue Solar SpA & Parque Fotovoltaico Peldehue Solar & Colina & $\mathrm{RM}$ & 120 \\
\hline Inversiones Iquique Solar $\mathrm{SpA}$ & Parque Iquique Solar & Pozo Almonte & Tarapacá & 120 \\
\hline Inversiones Frontera Solar SpA & Parque Frontera Solar & María Elena & Antofagasta & 105 \\
\hline
\end{tabular}

La Figura 1 muestra la distribución porcentual de ERNC, con predominio de la energía solar fotovoltaica, con $2.714,43 \mathrm{MW}(48,70 \%)$. seguida de la eólica con 1.793,46 MW $(32,17 \%)$, ubicada principalmente en el SEN, biomasa, minihidráulica con 508,96 MW $(9,13 \%)$ y geotermia con 48,47 MW (menor al 1\%).

La Figura 2 muestra cómo ha crecido considerablemente la potencia instalada de generación eléctrica basada en energía fotovoltaica. Al día de hoy, un incremento del 382,27\% más que el año 2015 de 711,04 MW. Cabe destacar el año 2017, donde se produjo la mayor cantidad de potencia instalada de 752,33 MW, al año siguiente se instalaron 475,15 MW y el año 2019 un desarrollo menor, pero no menos considerable, de 362,78 MW. La potencia acumulada al año 2020 que pueden generar las plantas existentes es de 2.718,03 MW.

La Tabla I muestra la capacidad instalada de las diez plantas fotovoltaicas más grandes a marzo de 2020 [16], destacando la planta solar El Romero con 196 MW (11.30\% del total). En segundo lugar, Luz del Norte con 141,04 MW. y en último lugar Llanos del Campos con 101,02 MW. La ubicación de todos los proyectos en el norte del país se justifica por:

- La ubicación de éstos presenta resultados favorables de radiación solar corroborados por datos de radiación de varias bases de datos certificadas [17], [18].

- El sitio está cerca de las líneas de distribución y subestaciones existentes.

- Las condiciones topográficas son apropiadas, propicias para la implementación de sistemas fotovoltaicos.

- Debido a las características propias del terreno donde se emplazan los proyectos, no se requiere la corta de flora y/o vegetación. 
TABLA III: Proyectos fotovoltaicos aprobados, "Proyectos de generación en el SEIA", marzo 2020, Generadoras de Chile.

\begin{tabular}{lllll}
\hline Propietario & Nombre & Comuna & Región & Potencia (MW) \\
\hline GR Notro SpA & Planta Fotovoltaica Lockma & Antofagasta & Antofagasta & 9 \\
GR Tara SpA & Planta Fotovoltaica Ckilir & Antofagasta & Antofagasta & 9 \\
MVC Solar 27 SpA & Parque Fotovoltaico Corcolenes & Los Ángeles & Biobío & 9 \\
Mandinga Solar SpA & Central Solar Fotovoltaica Mandinga & Melipilla & RM & 9 \\
Andina Solar 3 SpA & Parque Fotovoltaico Bollenar & Melipilla & RM & 9 \\
Torino Solar SpA & Planta Fotovoltaica Torino Solar & Teno & Maule & 9 \\
GR Pitao SpA & Planta Fotovoltaica Teno Uno 9 MW & Teno & Maule & 9 \\
Milán Solar SpA & Planta Fotovoltaica Milán Solar & Teno & Maule & 7 \\
\hline
\end{tabular}

TABLA IV: Los 10 proyectos más importantes de parques fotovoltaicos en construcción, "Proyectos en Construcción Histórico", marzo 2020, Energía Abierta.

\begin{tabular}{lllll}
\hline Owner & Name & Region & Power (MW) & Commissioning \\
\hline Enel Green Power del Sur SpA & Campos del Sol & Atacama & 381 & Nov-20 \\
Sol del Desierto SpA & Planta FV Sol del Desierto Fase I & Antofagasta & 175 & Dic-20 \\
Acciona Energía Chile SpA & Parque FV Malgarida II & Atacama & 162,70 & Mar-21 \\
TSGF SPA & Santa Isabel Etapa I & Antofagasta & 158,70 & Oct-20 \\
Atacama Solar S.A. & Atacama Solar II & Tarapacá & 150 & Sep-20 \\
AR Escondido SpA & Río Escondido & Atacama & 145 & Oct-20 \\
GPG Solar Chile 2017 SpA & Parque Fotovoltaico San Pedro & Antofagasta & 106 & Sep-20 \\
Parque Eólico Quillagua SpA & Parque Solar Fotovoltaico Nuevo Quillagua & Tarapacá & 100 & May-20 \\
Engie Energía Chile S.A. & Parque Solar Capricornio & Antofagasta & 87,90 & Ago-20 \\
Austrian Solar Chile Seis SpA & Parque Fotovoltaico La Huella & Coquimbo & 84 & Oct-20 \\
\hline
\end{tabular}

\section{Lo Que Sigue}

En este apartado se presentan los proyectos en construcción, aprobados y en clasificación, destinados a contribuir a satisfacer el constante incremento registrado en la demanda energética del país, lo que permitirá entregar energía limpia al Sistema Eléctrico Nacional (SEN), aumentando así la cantidad de ERNC a la matriz energética.

La Tabla II muestra los parques solares superiores a 100 MW en estado de análisis, a la espera de la aprobación de su Declaración de Impacto Ambiental (DIA) por parte de la SEIA a marzo de 2020 [19].

Son 26 proyectos que en conjunto suman 4.944,90 MW, siendo el de mayor capacidad el Parque Fotovoltaico Alfa Solar de Pleiades S.A, ubicado en María Elena, Región de Antofagasta con 729 MW. En segundo lugar, Planta Fotovoltaica Inti Pacha de Colbún S.A., ubicada en el mismo lugar con 719 MW.

La Tabla III muestra los parques solares fotovoltaicos aprobados por el Sistema de Evaluación de Impacto Ambiental (SEIA), a marzo de 2020 [19], que en conjunto suman un total de 70 MW. La mayoría son proyectos de 9 MW. Hay cuatro proyectos en las regiones sur de Chile, tres en la región del Maule y uno en la región del Biobío.

La Tabla IV muestra los diez proyectos de parques fotovoltaicos más importantes en construcción, actualizados a marzo de 2020 [20]. La potencia que estos sistemas añadirán al SEN asciende a $1.610,10 \mathrm{MW}$.

La mayor capacidad es Campos del Sol de Enel Green Power del Sur SpA con 381 MW. Como se puede ver en todas las tablas, los lugares con más proyectos son las regiones de Antofagasta y Atacama, ya que estas cuentas tienen las tasas de irradiación global horizontal más altas, ver Figura 3.
TABLA V: Características técnicas del panel solar JAP6-72 320 4BB.

\begin{tabular}{lr}
\hline Característica & Valor \\
\hline Voltaje en circuito abierto, $V_{o c}$ & $46,22 \mathrm{~V}$ \\
Voltaje a potencia máxima, $V_{m p}$ & $37,38 \mathrm{~V}$ \\
Corriente en corto circuito, $I_{s c}$ & $9,06 \mathrm{~A}$ \\
Corriente a potencia máxima, $I_{m p}$ & $8,56 \mathrm{~A}$ \\
Temperatura de operación & $-40^{\circ} \mathrm{C} /+85^{\circ} \mathrm{C}$ \\
Máximo voltaje de circuito abierto del sistema & $1000 \mathrm{~V}$ \\
Temperatura nominal de operación de la celda & $45 \pm 2^{\circ} \mathrm{C}$ \\
Coeficiente de temperatura $I_{s c}$ & $0.058 \% /{ }^{\circ} \mathrm{C}$ \\
Coeficiente de temperatura $V_{o c}$ & $-0.33 \% /{ }^{\circ} \mathrm{C}$ \\
Coeficiente de temperatura - potencia & $-0.41 \% /{ }^{\circ} \mathrm{C}$ \\
\hline
\end{tabular}

\section{La Mayor Planta Fotovoltaica}

En este apartado se muestran las principales características técnicas de la planta de mayor potencia actualmente en funcionamiento.

\section{V-A. Planta Fotovoltaica El Romero}

El Romero de ACCIONA Energía, es el parque fotovoltaico de mayor potencia que opera actualmente en el SEN, con una potencia instalada de $246 \mathrm{MWp}$. Se ubica a $8 \mathrm{~km}$ al sur de la localidad de Cachiyuyo, región de Atacama. Los paneles fotovoltaicos utilizados en el proyecto son del tipo silicio policristalino:

- JAP6-72 315 4BB (342.840 unidades de $315 \mathrm{~kW}$ ).

- JAP6-72 320 4BB (343.070 unidades de $320 \mathrm{~kW})$.

- 4BB-HR + 315P (90.320 unidades de $315 \mathrm{~kW})$.

Las características técnicas más relevantes están mostradas en la Tabla V. El proyecto consiste de 776.230 paneles solares y cubre un área de $2.800 .000 \mathrm{~m} 2$. Los inversores trifásicos 
usados son de la marca ABB de $400 \mathrm{~V}$, modelo PVS800 y son 180 unidades de $1.200 \mathrm{~kW}$ cada uno.

\section{CONCLUSIONES}

Este artículo ha presentado la capacidad de la matriz energética, que opera en base a energías renovables convencionales y no convencionales. Además, se ha constatado el estado de la energía solar fotovoltaica desde 2015 a la actualidad en Chile, detallando las plantas de mayor capacidad que se encuentran operando en el sistema eléctrico nacional, así como también los proyectos más significativos en construcción, homologado y en clasificación, que en conjunto representan un incremento de la matriz energética de 6.625 MW, ubicada mayoritariamente en las regiones de Antofagasta y Atacama del norte del país, debido a sus altas tasas de radiación. También se ha descrito la planta más grande de América Latina y sus características técnicas. Todo ello en concordancia con la política energética de Chile, que como meta para 2050 al menos el $70 \%$ de la generación eléctrica nacional debe provenir de energías renovables, y los autores pueden concluir que la energía fotovoltaica seguirá en aumento, como se muestra en los gráficos, y se espera que sea el principal participante en la generación eléctrica.

\section{AGRADECIMIENTOS}

Los autores agradecen el apoyo económico del Proyecto de Investigación FONDECYT Regular 1191028, al FONDAP SERC Chile 15110019 y el apoyo del Fondo Concursable para el Emprendimiento de la Investigación Científica de Estudiantes de Pregrado al estudiante Richard Arancibia de la Universidad de Talca.

\section{REFERENCIAS}

[1] T. V. Myasnikova, A. A. Kirillova, S. P. Ivanova, O. V. Sveklova, and O. A. Nadezhdina, "Simulation of solar energy photovoltaic conversion," in 2020 International Youth Conference on Radio Electronics, Electrical and Power Engineering (REEPE), 2020, pp. 1-4.

[10] P. Magazine, "https://bit.ly/3pfhz5x," Chile, el paraíso de la energía solar, Tech. Rep.
[2] A. Pino, F. J. P. Lucena, and J. G. Macho, "Economic analysis for solar energy integration in a microbrewery," in 2019 International Conference on Smart Energy Systems and Technologies (SEST), 2019, pp. 1-6.

[3] F. M. Guangul and G. T. Chala, "Solar energy as renewable energy source: Swot analysis," in 2019 4th MEC International Conference on Big Data and Smart City (ICBDSC), 2019, pp. 1-5.

[4] Enelx, "https://bit.ly/3a0git4," Tech. Rep.

[5] G. Jimenez-Estevez, R. Palma-Behnke, R. Roman Latorre, and L. Moran, "Heat and dust: The solar energy challenge in chile," IEEE Power and Energy Magazine, vol. 13, no. 2, pp. 71-77, 2015.

[6] M. Ahsan, N. Ahmad, and H. M. W. Badar, "Simulation of solar angles for maximizing efficiency of solar thermal collectors," in 2019 3rd International Conference on Energy Conservation and Efficiency (ICECE), 2019, pp. 1-5.

[7] G. Gajardo-Miranda, W. Puchi, J. L. Venegas, N. Risso, and F. Salgado, "A sustainability-based comparison between photovoltaics and concentrating solar power in chile," in 2019 IEEE CHILEAN Conference on Electrical, Electronics Engineering, Information and Communication Technologies (CHILECON), 2019, pp. 1-6.

[8] M. Najibullah, M. M. Mu Shabbir, and M. Y. Ali, "Solar based energy saving smart industrial exhaust fan," in 2019 22nd International Conference on Computer and Information Technology (ICCIT), 2019, pp. 1-5.

[9] E. Chile, "https://bit.ly/39d7wza," Aprende con Energía, Tech. Rep.

[11] P. Ramirez-Del-Barrio, P. Mendoza-Araya, F. Valencia, G. León, L. Cornejo-Ponce, M. Montedonico, and G. Jiménez-Estévez, "Sustainable development through the use of solar energy for productive processes: The ayllu solar project," in 2017 IEEE Global Humanitarian Technology Conference (GHTC), 2017, pp. 1-8.

[12] CNE, "Comisión nacional de energía," Tech. Rep., https://www.cne.cl/.

[13] CNE, "Reportes energéticos: mensual, ernc y financiero," Comisión Nacional de Energía, Tech. Rep., https://www.cne.cl/.

[14] Y. Simsek, C. Mata-Torres, T. Urmee, P. A. Bahri, and R. Escobar, "An assessment of incentives combination for solar energy technologies-a case study for chile," in 2018 International Conference on Smart Green Technology in Electrical and Information Systems (ICSGTEIS), 2018, pp. 29-34.

[15] G. G. Energy, "Sistemas fotovoltaicos y bombeo solar," June, 2018, Tech. Rep., https://ggechile.cl/.

[16] CNE, "Capacidad instalada por sistema eléctrico nacional," Comisión Nacional de Energía, April 2020, Tech. Rep., https://www.cne.cl/.

[17] Chile, "Global solar atlas," Tech. Rep., https://globalsolaratlas.info/download/chile.

[18] M. de Energía, "Explorador solar," Tech. Rep., http://www.minenergia.cl/exploradorsolar/.

[19] G. de Chile, "Boletín mercado eléctrico sector generación," Tech. Rep., April 2020.

[20] E. Abierta, "Proyecto de generación en etapa de construcción histórico," Tech. Rep., April 2020. 